Association for Information Systems AIS Electronic Library (AISeL)

PACIS 2006 Proceedings

Pacific Asia Conference on Information Systems

(PACIS)

2006

\title{
Using Peer-to-Peer Technology for Knowledge Sharing in Communities of Practices
}

Chen-Ya Wang

Lunghwa University of Science and Technology, d91003@im.ntu.edu.tw

Hsin-Yi Yang

Lunghwa University of Science and Technology, hsyyang@hotmail.com

Seng-cho T. Chou

Lunghwa University of Science and Technology, chou@ntu.edu.tw

Follow this and additional works at: http://aisel.aisnet.org/pacis2006

\section{Recommended Citation}

Wang, Chen-Ya; Yang, Hsin-Yi; and Chou, Seng-cho T., "Using Peer-to-Peer Technology for Knowledge Sharing in Communities of Practices" (2006). PACIS 2006 Proceedings. 67.

http://aisel.aisnet.org/pacis2006/67

This material is brought to you by the Pacific Asia Conference on Information Systems (PACIS) at AIS Electronic Library (AISeL). It has been accepted for inclusion in PACIS 2006 Proceedings by an authorized administrator of AIS Electronic Library (AISeL). For more information, please contact elibrary@aisnet.org. 


\title{
Using Peer-to-Peer Technology for Knowledge Sharing in Communities of Practices
}

\author{
Chen-Ya Wang ${ }^{1,2} \quad$ Hsin-Yi Yang ${ }^{1}$ \\ Department of Information Management ${ }^{1}$ \\ Seng-cho T. Chou ${ }^{1}$ \\ National Taiwan University \\ Department of Information Management ${ }^{2}$ \\ Lunghwa University of Science and Technology \\ d91003@im.ntu.edu.tw \\ hsyyang@hotmail.com \\ chou@ntu.edu.tw
}

\begin{abstract}
Communities of Practices (CoPs) are informal structures within organizations that bind people together through informal relationships and the sharing of expertise and experience. As such, they are effective tools for the creation and sharing of organizational knowledge, and, increasingly, organizations are adopting them as part of their knowledge management strategies. In this paper, we examine the knowledge sharing characteristics and roles of CoPs and develop a peer-to-peer knowledge sharing architecture that matches the behavioral characteristics of the members of the CoPs. We also propose a peer-to-peer knowledge sharing tool called KTella that enables members of CoPs to voluntarily share and retrieve knowledge more effectively.
\end{abstract}

Keywords: Knowledge Sharing, Communities of Practices (CoPs), Peer-to-Peer (P2P)

\section{Introduction}

Knowledge assets are a critical strategic resource and can provide organizations with a competitive advantage. Consequently, in recent years, research into and application of knowledge management (KM) techniques have become very important topics in management literature. Generally, knowledge can be categorized as either explicit knowledge or tacit knowledge (Nonaka et al. 1995). Most organizations focus on managing explicit knowledge well and try to capture tacit knowledge embedded in the experienced and skilled people as much as possible. Many approaches have been developed to help organizations create, capture, store, share, and apply knowledge. Often knowledge sharing is the core intent of a KM initiative, so it has become an important theoretical and practical problem (Scholl et al. 2004). By emphasizing interaction through social networks (person-to-person relationships), community-based knowledge sharing has become one of the most effective tools among a variety of approaches (Wenger et al. 2000; Koh et al. 2004). One reason that CoPs play an important role in knowledge management is that knowledge cannot be separated from its context (Pan et al. 2003). Despite their formal structures and policies, more and more organizations are developing CoPs as strategic tools for knowledge creation and sharing within an organization and even across organizational boundaries.

KM research can be divided into two categories: human-oriented and technologyoriented research (Maier et al. 2003). It is widely recognized that technology can support knowledge management activities and can often be an enabler of knowledge management. 
Traditional knowledge management technological architectures (e.g., Enterprise Knowledge Portal developed by Lotus Notes) are usually centralized, which makes it easy to control, organize, and store knowledge. In addition, they usually have a unique, simple point of access to incorporate the knowledge of the members from different organizational units (Mangisengi et al. 2003). Once an organization's members post their knowledge, they become detached from it, and lose control over who can access and use it. As a result, members are more likely to store their draft notes and working documents on their local repositories, rather than in a central knowledge management system. Many knowledge management systems are shunned by users, because they are not willing to update knowledge in the systems. One reason that people do not contribute to knowledge management systems continuously is that the technological architecture of such systems does not match human social behavior and work processes (Kwan et al. 2003; Maier et al. 2003; Scholl et al. 2004). Another reason is that knowledge workers do not want to give up autonomy and anonymity (Mangisengi et al. 2003). The design of KM systems needs to be consistent with the social processes of organization cognition (Bonifacio et al. 2002). One difficulty in KM nowadays is how to bridge the gap between the technical architecture and human factors (i.e., behavior). Peer-to-peer technology enables users to actively share knowledge and information in a more flexible way. It fits well with a more loosely coupled information-sharing environment (e.g., virtual teams, B2B knowledge sharing) and is 'natural' and 'personal' to users (Woods 2001; Tsui 2001). Peer-to-peer technology can complement a centralized technological architecture to help CoP members share their knowledge and enhance their social interaction. In this paper, we propose a peer-to-peer architecture to support knowledge sharing in CoPs from a sociotechnical perspective that considers the perspectives of human behavior and technological architecture support. We have also developed a tool called KTella to facilitate knowledge sharing between the members of CoPs.

The remainder of this paper is organized as follows. In Section 2, we present a review of CoPs and discuss the characteristics of knowledge sharing. In Section 3, we introduce the KTella architecture that can support knowledge sharing within and among CoPs. Section 4 describes two application scenarios of KTella. Finally, in Section 5, we present our conclusions and indicate the direction of future work.

\section{Knowledge Sharing in Communities of Practices}

The concept of communities of practice (CoPs), which was introduced by Lave and Wenger (1991), describes informal groups of people who create, share, and leverage their knowledge and experience. CoPs are self-organizing structures with a collective purpose and are held in place by social relationships (Agresti, 2003). CoPs can strengthen the ties between people in the same professional group and extend the network to a larger group. Unlike conventional functional organizations, the members of CoPs usually come from different units of an organization, or even from different organizations (e.g., professional associations, groups of software developers, or skilled craft guilds.) and complement the function of formal units.

Intangible, tacit knowledge embedded in an organization's members is an asset that is not easy to capture. CoPs offer a practical mechanism to help their members share and internalize tacit knowledge. Furthermore, through CoPs, people can deepen their expertise by discussing work-related activities with others in their field. As well as 
enabling members to share existing knowledge, CoPs also provide opportunities for new knowledge creation. Many researchers note that CoPs appear to be a more effective tool for dealing with unstructured problems and knowledge sharing/creation than traditional and formal ways of structuring interaction in organizations (Wartburg et al. 2004; Lesser et al. 2001; Kankanhalli et al. 2003). Many organizations, such as Motorola, HP, IBM, Xerox, Ford, and Shell have adopted CoPs as a KM tool. To increase knowledge sharing, the exchange of insights and ideas, and the transfer of expertise and experience, we can view CoPs as engines of knowledge creation and sharing (Lesser et al. 2001). For successful CoP implementation, it is important to: 1) remove barriers to individual participation, 2) support and enrich the development of each individual's uniqueness within the context of the community, and 3) link that uniqueness with the community's purpose (Liedtka 1999; Ardichvili et al. 2003).

Knowledge cannot be easily separated from its context and its owner. Understanding the processes and mechanisms that enable members to share knowledge with their peers in CoPs is very important for CoP knowledge sharing (Pan et al. 2003). There is no doubt that information technology plays an important role in CoP activities. In the past few years, collaborative technologies such as e-mail, listservs, electronic forums, and electronic chat rooms have facilitated the development of CoPs whose members are not collocated. (Brown et al. 1991; Wasko et al. 2000). A great deal of KM research has found that CoPs enabled by online interactive technologies can function as strategies for bringing the human behavior side into the adoption of KM ( Liedtka 1999; Ardichvili et al. 2003). The technological supports for CoPs are traditionally centralized architectures that facilitate easy control and management of knowledge; however, they do not fit knowledge workers' behavior well. For example, people have to link to a portal to upload their knowledge objects and login to forums to post articles or discuss practices with other members. One requirement for a successful $\mathrm{CoP}$ is that its members feel comfortable when participating in a computer-mediated, Internet-based CoP (Ardichvili et al. 2003). Technical support should help members to select useful information from the mass of postings and knowledge objects.

A KM system's social and technological attributes determine the success of knowledge creation and sharing (Holshouse 1998). Existing knowledge sharing and creation approaches tend to focus too much on either social or technological issues. Peer-to-peer architecture integrates the socio-technical elements to support the KM activities of CoPs, while KTella provides a recommendation function which helps users more efficiently find required knowledge objects. We introduce the system concept and architecture of KTella in the following section.

\section{KTella P2P Knowledge Sharing System Architecture}

\subsection{System Concept}

Our design goal is to form a knowledge sharing community based on decentralized $\mathrm{P} 2 \mathrm{P}$ technology. The technological architecture of a P2P system, in which a peer is both a server and a client, is consistent with the role of the community, in which a member is both a knowledge provider and a consumer. Thus, we utilize a P2P file sharing system for knowledge sharing.

We propose a P2P knowledge sharing system, called KTella, based on the Gnutella 
P2P network. A peer is generally referred to as a servent (both a SERVer and a cliENT). Gnutella is a representative of the decentralized and unstructured P2P system for the sharing of files over the Internet. It was designed in the early 2000 as a replacement for Napster and has been used mainly for the dissemination of multimedia files. Its simple and robust characteristics have made it famous and popular. To share files on the Gnutella network, a participant (node A for example) starts with a networked computer that runs one of the Gnutella clients. Node A will then connect to another Gnutellaenabled networked computer (node B for example) and announce its existence to B. Node $\mathrm{B}$ will in turn announce to all its neighboring nodes (nodes $\mathrm{C}, \mathrm{D}$, and $\mathrm{E}$ for example) that $\mathrm{A}$ is alive. This pattern will continue recursively with each new level of nodes announcing to its neighbors that node A is alive. Once node A has announced its existence to the rest of the network, the user at this node can now query the contents of the data shared across the network. We propose some modifications to Gnutella to boost the effectiveness of blind search. Our idea is that each peer links directly to nodes which similar with. There is a higher probability that these similar peers have the closest matches to the query keywords. By using a similarity-weighted sum of the other users' ratings, collaborative filtering algorithms can predict a user rating for an item. We adopt rating mechanism to form peer group and to provide social filtering and recommendation to make knowledge sharing effective and efficiency.

KTella seeks to retain the simple, robust, and fully decentralized nature of Gnutella, while improving its efficiency. Locating content efficiently in a decentralized P2P system is a challenging problem. Efficiency in knowledge sharing means to provide the right information to the right people at the right time. We use a rating method to support recommendations, and peer clustering to make knowledge sharing efficiently. KTella provides the functionality to support knowledge sharing activities and promote knowledge sharing in the form of document sharing, ideas and experience sharing, and rating sharing.

KTella has five main functions: knowledge searching, knowledge publishing and forwarding, peer clustering, knowledge recommendation, and instant message. A KTella user is both a knowledge consumer and a knowledge provider. As a consumer, the user searches in KTella for knowledge objects that he needs or is interested in. On the other hand, as a provider, he also publishes and forwards knowledge objects to others. Peer clustering finds other users with similar knowledge domains, and acts as a social filter to make searching, publishing, and forwarding knowledge objects more efficient. The knowledge recommendation function provides a predicted rating of a sharable knowledge object (SKO) to recommend to users, while the instant message function provides a channel for communication between KTella users.

KTella consists of two components: a knowledge sharing component and a P2P network component. The former facilitates knowledge sharing between members in a community, while the latter deals with a set of descriptors used for communication between servents and sets rules for inter-servent descriptor exchange. In the following sections, we describe these two components of KTella. Before introducing our system, however, we define some terms used in the KTella system.

Colleague Group: Peers in relevant organizational divisions are combined in a colleague group. Relevant divisions may be finance departments in different countries, or departments in the same college. Their relationships are explicit in an organization's 
architecture. Each peer has a colleague group list to keep a record of his/her colleague peers.

Companion Group: Peers with the same interests are included in the companion group. Each peer has a companion group list to keep a record of his/her companion peers. Since a peer's interests may change, the companion peers list will update dynamically according to the peer's similarity to other potential companion peers.

Knowledge Peer: A knowledge peer is in either a colleague group or a companion group.

Information Peer: An information peer does not belong to a colleague group, or a companion group.

Sharable Knowledge Object (SKO): This is a knowledge object that a peer wants to share with others. It could be a document that a knowledge worker wrote to describe his experience, or a document that is useful and related to his work.

Relevancy Rating: This represents the extent that the SKO is relevant to the knowledge worker's domain.

Utility Rating: This represents the extent that the SKO is useful and/or of interest to the knowledge worker.

In addition, KTella makes the following assumptions:

(1) Most knowledge workers are servents. Since KTella is a knowledge sharing system, most knowledge workers need to connect to KTella.

(2) A user will give ratings on an SKO after browsing. These ratings are used to form a peer group in order to find companions and facilitate social filtering and recommendations.

(3) Each peer stores SKOs with high utility ratings. When a user gives a high rating to a document, it means that it is useful or interesting to him. We assume that it will be stored.

\subsection{KTella Algorithms}

\subsubsection{Definition}

First, we present some definitions that will be used in the following sections. There is a set $N$ of node is in KTella and each node $n \in N$ has:

NodeID ( $n)$ : a unique id.

$P$ : a set of colleague groups.

$q$ : a companion group.

$N p$ : a set of nodes in colleague groups, where $N p=n \in p \square p \in P$.

$N q$ : a set of nodes in companion group, where $N q=n € q$.

$N k$ : a set knowledge peers, where $N k=\{N p \square N q$.

$N i$ : a set information peers, where $N i=N-N k$.

$V u t i l \sigma$ : the utility vote $n$ gives to $\operatorname{SKO} \sigma$.

Vrel $\sigma$ : the relevancy vote $n$ gives to $\mathrm{SKO} \sigma$.

$R n i$ : relevancy to node $i$, where $i$ is other nodes in KTella

$$
\mathrm{Rni}=\frac{\sum_{\dot{0}}\left(v_{n, \mathrm{o}}-\bar{v}_{n}\right)\left(v_{i, \mathrm{o}}-\bar{v}_{i}\right)}{\sqrt{\sum_{j}\left(v_{n, j}-\bar{v}_{n}\right)^{2} \sum_{j}\left(v_{i, j}-\bar{v}_{i}\right)^{2}}}
$$

where the summations over $\sigma$ are over the SKOs for which both nodes $n$ and $i$ recorded relevancy ratings.

There is a set of $O$ of SKOs is shared in KTella and each SKO $\sigma \in O$ has: 
ObjectID $(\sigma)$ : a unique id

$K \sigma$ : a set of keywords associated with

\subsubsection{SKO Search Algorithm}

$O K$ : a set of matching SKOs, where $O K=\{\sigma \square K \subseteq K \sigma, \sigma \in O$ \}.That is, $O K$ is the set of SKOs that can be described by $K$.

Knowledge Peers Searching Phase: search $O K$ in Nk.

Information Peers Searching Phase: search $O K$ in $N i$.

When a user of node $n$ searches SKO with a set $K$ of keywords:

If $O K$ in Knowledge Peers Searching Phase $\neq \Phi$

Then user decides whether to go to Information Peers Searching Phase Else

go to Information Peers Searching Phase

End if

End of algorithm

\subsubsection{SKO Forward Algorithm}

When a node $n$ gives utility rating on $\mathrm{SKO} \sigma$ :

If $V u t i l \sigma>$ threshold

then

for each group $p$, where $p \subseteq P$ :

$n$ decides whether to forward $\sigma$ to nodes in $p$

for each node $q$ in $N q$

If Vrel $\sigma>$ threshold

KTella automatically forwards $\sigma$ to $N q$

End if End if

End of algorithm

\subsubsection{Peer Clustering Algorithm}

The algorithm uses the Pearson correlation coefficient to determine if two peers are relevant.

For each node $n:$
$\quad$ If $R n i>0$
then include $i$ in $N q$
Else $\quad$ excludes $i$ from $N q$
End if
End of algorithm

\subsection{System Architecture}

KTella is a P2P knowledge sharing system. Each computer requires setup the KTella client in order to run as a servent. By following KTella's network protocol, peers can communicate with each other and connect to the KTella network. The core of the KTella 
network protocol is comprised of a set of descriptors which are used for communication between servents and also sets rules for the inter-servents descriptors exchange. We modify some of descriptors adopted from Gnutella and define some new ones to meet the requirements of the knowledge sharing system. The protocol design is shown as Figure1.

Each servent is equipped with a local repository of SKOs, which is available to the KTella network for sharing with other servents. The goal is that most knowledge workers in an organization will run servents to provide SKOs and ratings on them to increase the number of knowledge objects and the ratings repository available to the KTella network as a whole. The system architecture of KTella is illustrated in Figure2. The implementation of KTella includes client and network protocol implementation.

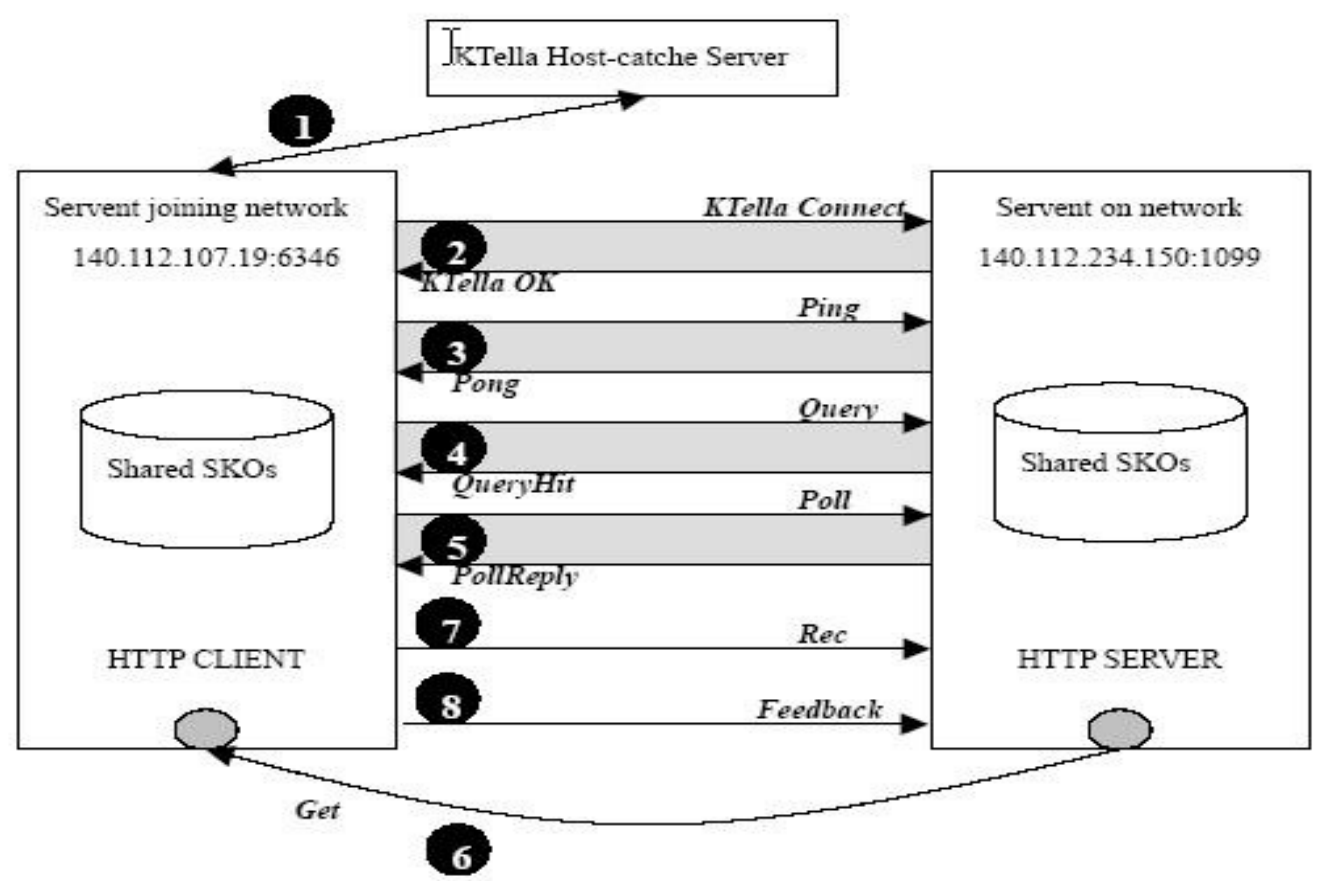

Figure1 KTella Network Protocol 


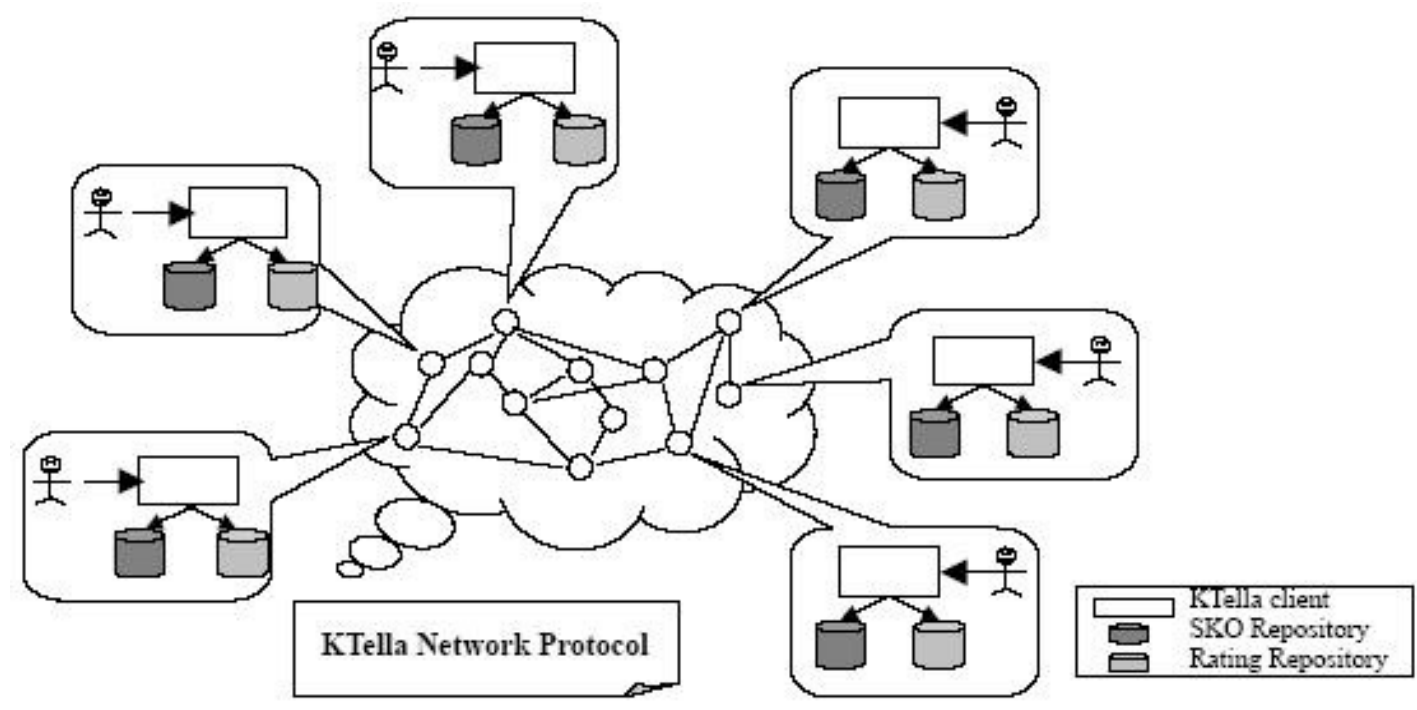

Figure2 KTella's System Architecture

Knowledge workers use the KTella clients to connect to the KTella network. Each client acts as a server to contribute SKOs and a client to receive them. KTella client is composed of a user interface module, a search module, an instant message module, a rating module, an SKO exchange module, a publishing module, a recommendation module, and a peer clustering module. The relationships among the modules are shown in Figure 3. KTella client operates through a user interface module to interact with other servents in the KTella network.

\section{- User Interface Module}

The user interface module acts as an intermediary between the user and other modules. The user interacts with the module when he searches for SKOs in KTella, publishes and forwards an SKO to KTella, gives ratings on an SKO, sends instant messages to others, or decides whether to download SKOs from other peers. The user interface module then interacts with the search module, publishing module, rating module, instant message module, and SKO exchange module to accomplish the user's task. Conversely when a servent receives a QueryHit from other servents, the user interface module presents related information to the user. 


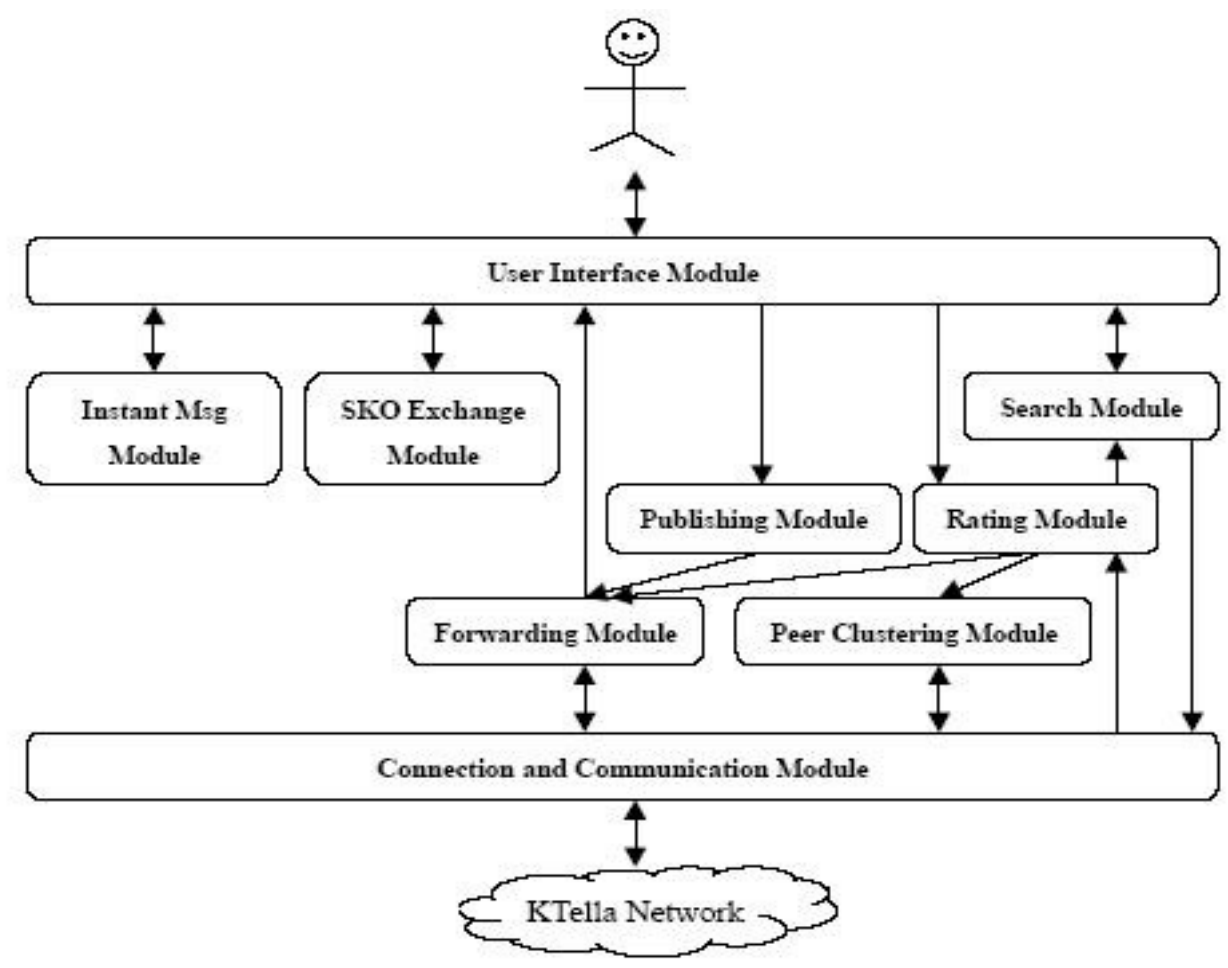

Figure3 KTella's Module Architecture

\section{- Search Module}

The search module deals with a user's search request from the user interface module to search target SKOs in KTella. When searching for an SKO, the user specifies keywords of the SKO's name and annotations as search criteria. The user interface module sends the search criteria to the search module, which encapsulates the criteria in a Query description and sends it to the connection and communication module to route into the KTella network.

The search module also deals with Query descriptions transmitted from the connection and communication module. First, it parses the search string in the Query description and then scans its SKO repository for any matching SKOs. If a target SKO is found, the search module sends back a QueryHit description containing the SKO and servent information. The annotations of the SKO are obtained from the SKO information database. If a target SKO is not found, the Query description will be dropped.

\section{- Publishing Module}

When a user considers that a document is an SKO, he/she sends it to KTella, and chooses which companion groups to forward it to. The user also assigns annotations to describe it to facilitate knowledge sharing. The publishing module moves the document to the SKO repository, which is a shared storage on a local disk, for other peers in KTella to access. The repository stores related SKO information in its database.

The publishing module then triggers the forwarding module to send the SKO to the colleague groups that the user chooses, as well as the companion group. Other peers in KTella can access the SKO by searching or having it forwarded to them.

\section{- Forwarding Module}

The rating and publishing modules trigger the forwarding module. When a user gives a rating on an SKO above the threshold, or sends an SKO to KTella, the forwarding 
module encapsulates the SKO information in a forwarding description and sends it to the connection and communication module to route it to its knowledge peers.

The forwarding module also deals with forwarding descriptions sent from other peers. When a servent receives a forwarding description, the forwarding module checks the forwarding log to avoid duplication. If a KTella servent receives the description for the first time, it keeps it in the forwarding log and triggers the user interface module to inform the user about the new SKO.

\section{- Rating Module}

The rating module deals with ratings on SKOs. When a user gives a rating on an SKO downloaded from another peer, the rating module stores it in the rating database. If the rating of the SKO is above the threshold we set, KTella keeps it in SKO repository and triggers the forwarding module. Otherwise, the SKO is deleted. The rating module then triggers the peer clustering module for group tuning. It also encapsulates its rating vector in a feedback description and sends it to servents who have sent PollReply descriptors.

When the rating module receives a Poll description from the connection and communication module, it looks up the rating database to check if there are ratings on the SKO that match the Poll description queries. If so, the rating module encapsulates the rating vector in a PollReply description and sends it to the connection and communication module to route into KTella network.

When a servent receives a QueryHit description, the rating module encapsulates Poll descriptions to poll other peers' ratings on the matching SKO. After receiving PollReply descriptions from other peers, the rating module calculates a predicted utility rating on each matching SKO. The predicted utility ratings are calculated by the modified collaborative filtering algorithm and used to complete the SKO predicted rating element in the result set of QueryHit description. The QueryHit description is then sent to the user interface module.

\section{- SKO Exchange Module}

The SKO exchange module deals with users' requests for file exchanges from the user interface module. When a user wants to download an SKO from other servents, the SKO exchange module has to download the SKO from the provider. It also deals with other peers' requests for SKO exchange.

\section{- Instant Message Module}

The instant message module deals with communication among peers. It sends instant messages to other peers and receives messages from them.

\section{- Peer Clustering Module}

When a user gives a rating on an SKO, the rating module triggers the peer clustering module to calculate its similarity to the ratings of other servents who have sent PollReply descriptors. When a servent receives the Feedback description, the rating module is also triggered to calculate its similarity with the servent who sends the description. The similarities among servents are calculated by Pearson's correlation coefficient. Whether or not a servent is included in a companion group list depends on its similarity to other members of the group.

\section{Application Scenarios}

In this section, we first present two scenarios to demonstrate our system, and then describe our utility's contribution. Each user of the KTella knowledge sharing system 
plays two main roles: knowledge provider and knowledge consumer, for which we provide separate scenarios.

\subsection{Knowledge Provider Scenario}

As a knowledge provider, the KTella client allows a user to publish any type of electronic resource that he/she considers as a knowledge object. The user selects the Share panel to publish an SKO, gives annotations and ratings on it, and chooses which groups to forward it to. This panel also shows information on SKOs he/she has published and the upload records of the SKOs. The screenshot is shown in Figure4.

After a user downloads an SKO from another peer, he/she is asked to give ratings and annotations on it and choose which groups to forward it to. This is illustrated in Figure5. Also, as knowledge providers, users share their professional opinions about recently downloaded SKOs.

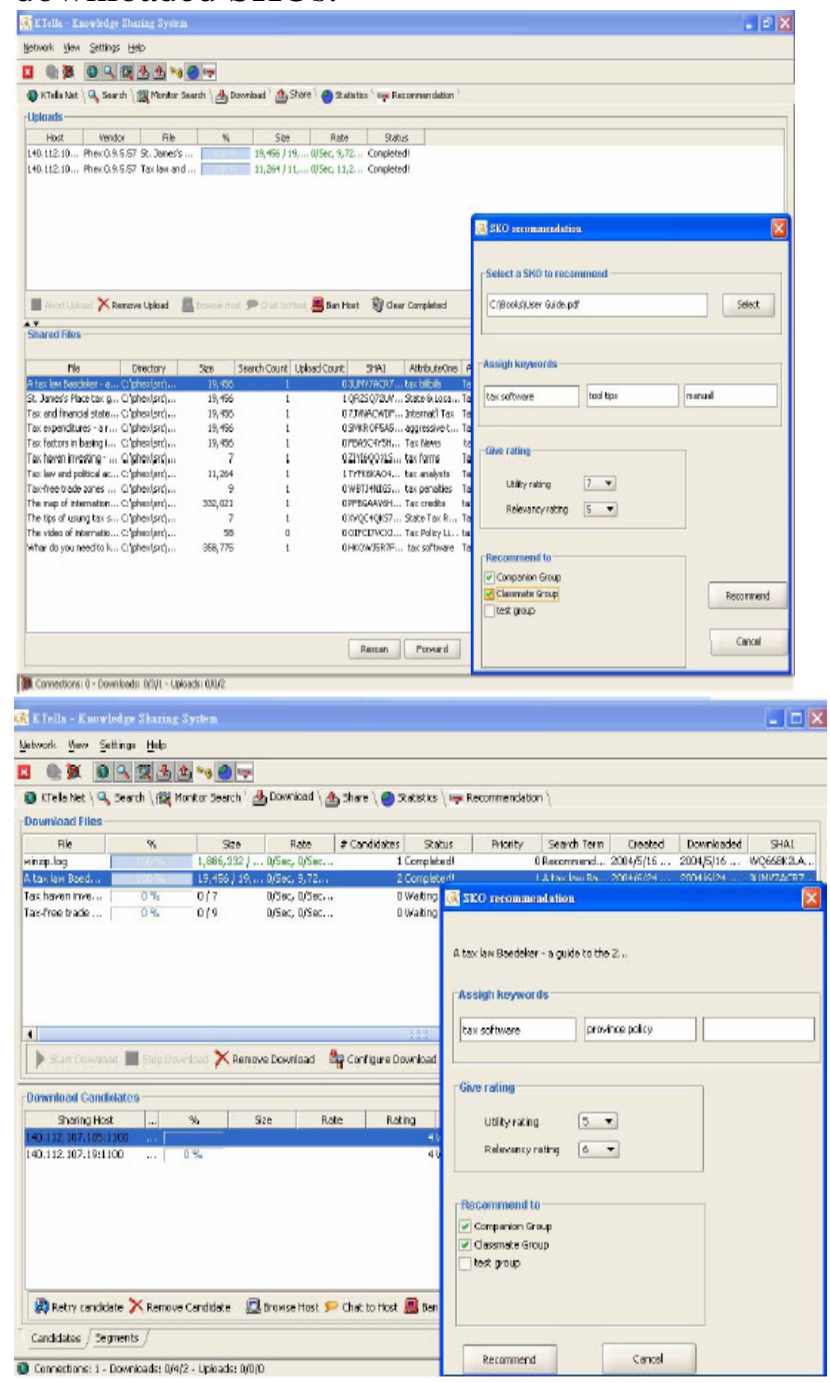

Figure4 A screenshot of SKO publishing Figure5 A screenshot of SKO forwarding

\subsection{Knowledge Consumer Scenario}


Knowledge consumer is the other role of the KTella system. As a knowledge consumer, the KTella client allows a user to search for SKOs from other peers. The user selects the Search tab and fills in the search criteria. Information about matching SKOs then appears on the right-hand side of the panel. It includes file information, sharing hosts, and predicted utility ratings to help the user decide whether to download, and where to download, the SKO. The user can also communicate directly with others by instant messaging to share ideas and experiences. In addition, he/she can browse others' SKO repositories to see if there are SKOs he/she needs. The screenshot is shown in Figure6.

As a knowledge consumer, the user receives SKOs forwarded from others. They are displayed in the Recommendation tab. The user can send instant messages with, and browse the SKO repository of, the forwarders. If he/she downloads the SKOs, the related information will be displayed in the Download tab. Figure7 is the screenshot of the Recommendation tab.

From the Download tab (Figure6), the user can also obtain information on SKOs downloaded from other peers. The search term column specifies the source of the SKO. It could be the search criteria for SKO matching or "Forwarding download", which means the SKO has been forwarded from others. For each SKO, the KTella client provides information on its download candidates. If a user wants to remove a download record, he/she has to give a utility rating and a relevancy rating on it. This mechanism enforces the rule of sharing ratings with other users.

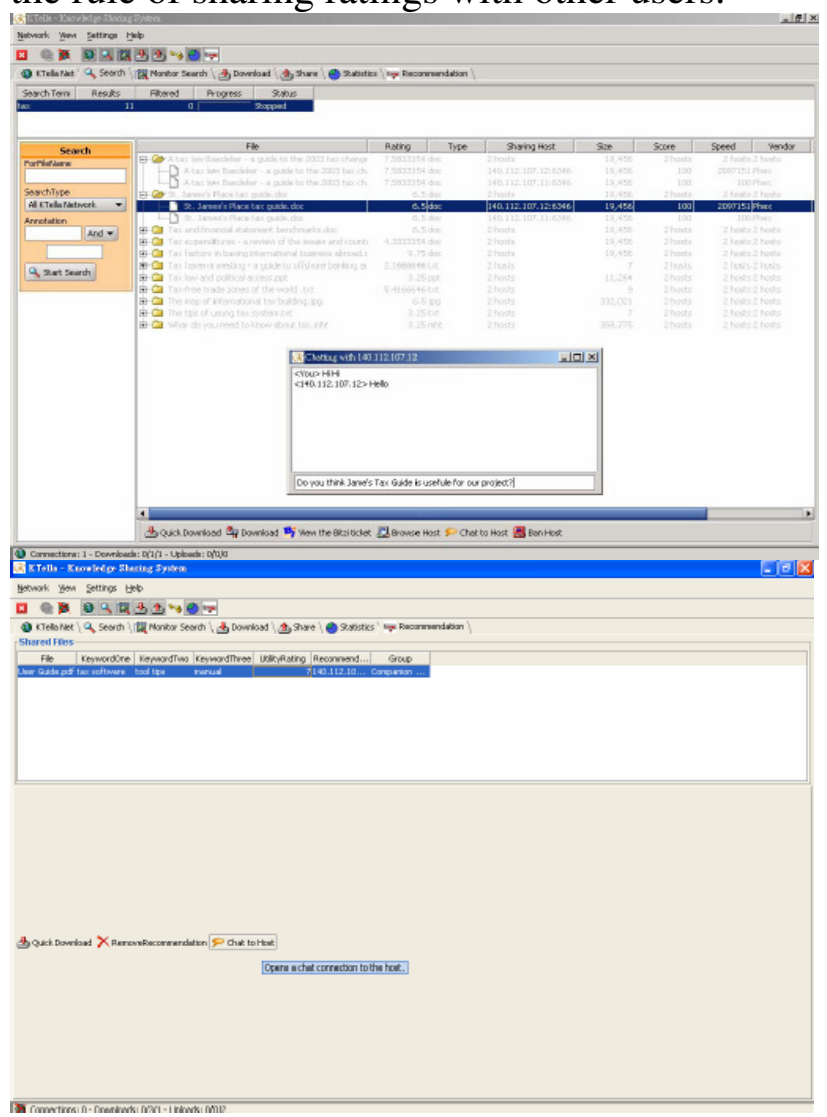

Figure6 A screenshot of SKO searching recommendation tab

Figure7 A screenshot of the

\subsection{Utility Discussion}


A knowledge sharing system empowers an individual knowledge worker by providing the tools to support and boost his/her knowledge sharing ability. The basic assumption is that if individuals are willing to share knowledge, they will be willing to use tools to share that knowledge. Thus, an effective knowledge sharing system should motivate users to access it and provide them with tools to facilitate knowledge sharing.

Kwok and Gao (2004) summarize and clarify the most salient motivational factors that influence the willingness, or tendency, of P2P network users to share knowledge. Their findings are used to add some new application features to the P2P community with particular focus on these motivational factors, in order to facilitate the knowledge-sharing process. The application features are: sub-community organization; individual identity and profile generation; contribution-reward mechanism; and reviews and peer recommendation.

KTella has the following application features:

1. Sub-community organization: KTella allows users to set up their own colleague groups. It also automatically forms companion groups for them. The formation of a subcommunity can provide community identity and a sense of belonging for an individual, so that he/she will be willing to do something beneficial for others.

2. Individual identity and profile generation: In KTella, each servent has a unique identity. This feature provides the fundamental element for building trusting relationships among members, and can induce effective knowledge sharing between them. The rating repository of each servent acts as a personal profile, presenting the interests and work domain of the user. According to the theory of interpersonal psychology, people tend to form affiliations with others who have similar interests.

3. Contribution-reward mechanism: To incorporate the reward features into the P2P knowledge sharing system, it is necessary to add a knowledge-tracking module to the application. KTella provides statistical information to evaluate an individual's contribution to the knowledge sharing community. An administrator can set up reward rules according to the statistical information.

4. Peer recommendation: This is used to help a user decide which member to interact with and share knowledge with on the basis of previous experience. Although KTella doesn't implement an exact peer recommendation feature, its peer clustering functionality provides a similar mechanism. In KTella, knowledge peers have a high priority to interact with each peer in the knowledge searching and knowledge forwarding processes.

KTella has the following basic knowledge sharing functions and an additional mechanism to make knowledge sharing effective and efficient.

1. Contributing

KTella allows a user to contribute any type of electronic resource as an SKO. Annotations of the SKO act as metadata for retrieval. After a user contributes his/her knowledge, KTella automatically forwards the SKO to others who may need it. This mechanism makes contributing knowledge more effective.

2. Searching

KTella allows a user to search for SKOs by using keywords and annotations and by browsing other peers' SKO repositories. KTella's search algorithm first searches the user's knowledge peers, because the probability that they will have matching SKOs is high. This mechanism makes searching for knowledge more efficient. In addition, KTella 
provides a predicted rating on each matching SKO, which helps the user decide which SKO to download.

3. Companion finding

KTella finds companion groups for a user. This allows knowledge and experience to be shared among people who otherwise would never know each other. Organizations can avoid repeating mistakes, duplicating efforts, and wasting resources because members of the organization know each other's work. This also benefits individuals by avoiding redundant efforts and facilitates better achievements.

4. Information filtering

In KTella, each user has a sub-community that provides a social filtering mechanism. In the knowledge searching and dissemination process, the sub-community filters in relevant knowledge and filters out irrelevant knowledge. This makes knowledge sharing effective and efficient.

\section{Conclusions}

Building CoPs is an effective method for creating and sharing organizational knowledge. Unlike centralized knowledge management approaches, peer-to peer architecture is a knowledge management option that matches human behavior with working processes. In a centralized system, people always have to upload their knowledge objects separately from their working process. P2P systems, on the other hand, provide a feature whereby people can save their knowledge objects in their own knowledge repositories when doing their work and do not need to do additional effort. In this paper, we have proposed a P2P knowledge sharing architecture from the human behavior and technical perspectives. We have also presented a system called KTella, based on the Gnutella P2P network. KTella seeks to retain the simple, robust, and fully decentralized nature of Gnutella, while providing a knowledge sharing functionality.

Specifically, KTella forms a knowledge sharing environment with multiple knowledge repositories, and enables knowledge to be produced in different formats by different producers at different functional levels. The roles of knowledge generation and codification are conducted by the same knowledge worker and contextual information is naturally embedded. Adopting a P2P network architecture makes the social form of organizations similar to a technology architecture so that the knowledge sharing process is more natural and, therefore, more acceptable to users.

We have incorporated a rating method in the P2P knowledge sharing system. Users are asked to give relevancy and utility ratings for SKOs, which are used for knowledge recommendation and peer clustering. To predict the utility ratings, we modify collaborative filtering for knowledge recommendation. This assists a user in making decisions about which SKOs to download. Peer clustering also forms sub-communities and finds companion groups for each user. The sub-community acts as a social filtering mechanism. Companion finding avoids duplicating efforts and wasting an organization's resources.

We believe that KTella can empower knowledge workers by providing them with the tools and environment to facilitate knowledge sharing. Meanwhile, the application features of KTella motivate knowledge workers to use it for knowledge sharing. KTella promotes knowledge sharing in the form of document sharing, ideas and experience 
sharing, and professional judgment sharing and provides powerful tools to support and boost more knowledge sharing activities.

KTella should be deployed in organizations. Since knowledge sharing is a continuous and long term process, it takes time to observe its evolution and incrementally tune the system to boost performance. Due to the time limitation, we have not completely deployed it in our current research. We will further examine the usefulness of KTella by simulation or experiment. Future deployment will be carried out within the NTU intranets.

In the future, we will extend Ktella by deploying a central server within the P2P network to deal with high volumes of network traffic. This extension will allow developers to add infrastructure and provide special features for knowledge sharing activities. Ontology technology could also be adopted by our knowledge sharing system. The goal is to capture domain knowledge in a generic way and provide a commonly agreed understanding of a domain, which may be reused and shared across applications and groups. Building such an ontology for a knowledge sharing system would make the system much more powerful.

\section{References}

Agresti, W.W. "Tailoring IT Support to Communities of Practice," IT Professional (5:6), 2003, pp. 24-28.

Ardichvili, A., Page, V., and Wentling, T. "Motivation and Barriers to Participation in Virtual Knowledge-Sharing Communities of Practice," Journal of Knowledge Management (7:1), 2003, pp. 64-77.

Bonifacio, M., Cuel, R., Mameli, G., and Nori, M. "A Peer-to-Peer Architecture for Distributed Knowledge Management, in Proceedings of the 3rd International Symposium on Multi-Agent Systems, Large Complex Systems, and E-Businesses MALCEB, 2002.

Brown, J.S., and Duguid, P., "Organizational Learning and Communities-of-Practice: Toward a United View of Working, Learning, and Innovation," Organization Science (2:1), 1991, pp. 40-57.

Holsthouse, D. "Knowledge Research Issues," California Management Review (40:3), 1998, pp. 277-280.

Iverson, J. O., and McPhee, R. D. "Knowledge Management in Communities of Practice," Management Communication Quarterly (16:2), 2002, pp. 259-266.

Kankanhalli, A., Tanudidjaja, F., Sutano, J., and Tan, B., "The role of IT in successful knowledge management initiatives," Communications of the ACM (46:9), 2003, pp. 69-73.

Koh, J., and Kim, Y. G. "Knowledge Sharing in Virtual Communities: an E-Business Perspective," Expert Systems with Applications (26:2), 2004, pp. 155-166.

Kwan, M. M., and Balasubramanian, P. "KnowledgeScope: Managing Knowledge in Context," Decision Support Systems (35:4), 2003, pp. 467-486.

Kwok J. S.H., and Gao, S. "Knowledge Sharing Community in P2P Network: a Study of Motivational Perspective," Journal of Knowledge Management (8:1), 2004, pp. 94102.

Lave, J., and Wenger, E. Situated Learning, Cambridge University Press, Cambridge, UK, 1991.

Lesser, E. L., and Storck, J. "Communities of Practice and Organizational Performance," IBM Systems Journal (40:4), 2001, pp. 831-841. 
Liedtka, J. "Linking Competitive Advantage with Communities of Practice," Journal of Management Inquiry (8:1), 1999, pp. 5-16.

Maier, R., and Remus, U. "Implementing Process-Oriented Knowledge Management Strategies," Journal of Knowledge Management (7:4), 2003, pp. 62-74.

Mangisengi, O., and Essmayr, W. "P2P Knowledge Management: an Investigation of the Technical Architecture and Main Process," in Proceedings 14th International Workshop on Database and Expert Systems Applications, 2003, pp. 787-791.

Nonaka, I., and Takeuchi, H. The Knowledge Creating Company, Oxford University Press, New York, 1995.

Pan, S. L., and Leidner, D. E. "Bridging Communities of Practice with Information Technology in Pursuit of Global Knowledge Sharing," Journal of Strategic Information Systems (12:1), 2003, pp. 71-88.

Rouser, K., and Dorsey, A. "Strong Threads of Learning-Knowledge Transfer within Communities of Practice," in IEMC '03. Managing Technologically Driven Organizations: The Human Side of Innovation and Change (2-4), 2003, pp. 141-145.

Scholl, W., König, C., Meyer, B., and Heisig, P. "The Future of Knowledge Management: an International Delphi Study," Journal of Knowledge Management (8:2), 2004, pp. 19-35.

Tsui, E. "Technologies for Personal and Peer-to-Peer (P2P) Knowledge Management, Technical Report," CSC Leading Edge Forum (LEF), Australia, 2001.

Wasko, M. M., and Faraj, S. "It Is What One Does: Why People Participate and Help Others in Electronic Communities of Practice," Journal of Strategic Information Systems (9), 2000, pp.155-173.

Wartburg, I. V., Rost, K., and Teichert, T. "The Creation of Social and Intellectual Capital in Virtual Communities of Practice," in the fifth European conference on Organizational Knowledge, Learning and Capabilities, 2004.

Wegner, E. C., and Synder, W. M. "Communities of Practice: The Organizational Frontier," Harvard Business Review (78:1), 2000, pp. 139-145.

Wiig, K. M. "What Future Knowledge Management Users May Expect, Journal of Knowledge Management (3:2), 1999, pp. 155-166.

Woods, E. "Knowledge Management and Peer-to-Peer Computing: Making Connections," KMWorld (10:9), 2001. 